\title{
Separase is a marker for prognosis and mitotic activity in breast cancer
}

\author{
Natalia Gurvits*,1, Eliisa Löyttyniemi ${ }^{2}$, Marjukka Nykänen ${ }^{3}$, Teijo Kuopio ${ }^{4}$, Pauliina Kronqvist ${ }^{1}$ \\ and Kati Talvinen ${ }^{1}$ \\ ${ }^{1}$ Institute of Biomedicine, Department of Pathology and Forensic Medicine, University of Turku and Turku University Hospital, \\ Turku 20510, Finland; ${ }^{2}$ Department of Biostatistics, University of Turku, Kiinamyllynkatu 10, Turku 20510, Finland; ${ }^{3}$ Department of \\ Pathology, Central Hospital of Central Finland, Keskussairaalantie 19, Jyväskylä 40620, Finland and ${ }^{4}$ Biological and Environmental \\ Science, University of Jyväskylä and Department of Pathology, Central Finland Health Care District, Keskussairaalantie 19, \\ Jyväskylä 40620, Finland
}

Background: Cancer cell proliferation is a critical feature in classifying and predicting the outcome of breast carcinoma. Separase has a central role in cell cycle progression in unleashing sister-chromatids at anaphase onset. Abnormally functioning separase is known to lead to chromosomal instability.

Methods: The study comprises 349 breast carcinoma patients treated in Central Hospital of Central Finland. The prognostic value, role as a proliferation marker and regulatory interactions of separase are evaluated by immunohistochemical and double- and triple-immunofluorescence (IF) detections based on complete clinical data and >22-year follow-up of the patient material.

Results: In our material, abnormal separase expression predicted doubled risk of breast cancer death $(P<0.001)$. Up to 11.3-year survival difference was observed when comparing patients with and without separase expressing cancer cell mitoses. Particularly, abnormal separase expression predicted impaired survival for luminal breast carcinoma $(P<0.001$, respectively). In multivariate analyses, abnormal separase expression showed independent prognostic value. The complex inhibitory interactions involving securin and cyclin B1 were investigated in double- and triple-IFs and revealed patient subgroups with aberrant regulation and expression patterns of separase.

Conclusions: In our experience, separase is a promising and clinically applicable proliferation marker. Separase expression shows strong and independent prognostic value and could be developed into a biomarker for treatment decisions in breast carcinoma, particularly defining prognostic subgroups among luminal carcinomas.

Separase (extra spindle poles-like 1), a cysteine protease and endopeptidase, has a central role in cell cycle progression in ensuring immaculate genetic inheritance. In the normal cell, activation of separase initiates anaphase at two diligently controlled consecutive events (Mora-Santos et al, 2011; Hellmuth et al, 2015; Meadows and Millar, 2015; Zhang and Pati, 2017). In the first event, separase triggers the cleavage of the $S c c 1 / \operatorname{Rad} 21$ subunit of cohesin, thus inactivating the complex. In the next event, separase participates in the final unleashing of the sister-chromatids at anaphase onset (Sun et al, 2009; Schöckel et al, 2011). Abnormally increased proteolytic activity of separase has been described to predispose the cell to uncontrolled centriole duplication, chromosomal missegregation and aneuploidy (Pati, 2008; Haaß et al, 2012). Owing to its central role in metaphase-anaphase transition, both premature and delayed activation of separase will result in chromosomal instability (Zhang and Pati, 2017).

Separase is tightly and in a mutually exclusive manner regulated by complex inhibitory interactions in pathways involving either securin (pituitary tumour-transforming gene 1 protein) or cyclin B1/CDK1 (G2 mitotic-specific cyclin-B1/cyclin-dependent kinase 1) (Zhang and Pati, 2017). In both of these pathways, the proteolytic activation of separase is dependent on ubiquitin-

*Correspondence: Dr N Gurvits; E-mail: natalia.gurvits@utu.fi

Received 5 May 2017; revised 29 June 2017; accepted 4 August 2017; published online 31 August 2017

(C) 2017 Cancer Research UK. All rights reserved 0007-0920/17 
dependent degradation of either securin or cyclin B1 (Hornig et al, 2002; Weizenegger et al, 2002; Holland and Taylor, 2006). Securin, an anaphase inhibitor, forms with separase a complex with ability to arrest the cell cycle at metaphase. Ubiquitin-dependent destruction of this link results in activation of separase triggering premature sister chromatid separation (Hornig et al, 2002). In addition, separase activity is controlled by cyclin B1 complex promoting dissociation of sister chromatid separation (Hellmuth et al, 2015). Both of these events are mediated by the anaphasepromoting complex/cyclosome (APC/C) promoting the destruction of securin and cyclin B1 (Shindo et al, 2012). Uncontrolled inhibition of separase activity by either securin or cyclin B1 will result in uncontrolled and premature segregation of the sister chromatids and hamper immaculate genetic inheritance, the cornerstone event of malignant development (Zhang et al, 2008).

Previously, overexpression and variation in subcellular localisation of separase have been reported in specific types of human malignancies, including breast carcinomas (Meyer et al, 2009; Zhang et al, 2014; Mukherjee et al, 2014b; Zhang and Pati, 2017). Although the role of separase as an oncogene and in promoting aneuploidy and genetic heterogeneity is well established (Zhang and Pati, 2017), the different expression patterns, associations with clinically applied proliferation markers or prognostic associations of separase have not previously been described in a large breast carcinoma material with long-term follow-up. In the present study, we demonstrate separase expression in a 349 breast carcinoma patients with a maximum of $>22$-year follow-up. Based on double- and triple-immunofluorescence (IF) stainings, we also describe the co-expressions and discuss the possible roles of securin and cyclin B1 in regulation of separase expression in breast carcinomas. In our experience, separase is a promising proliferation marker that could be applied into clinical use in histopathology. Moreover, in comparison with the established clinical prognosticators, separase expression showed strong and independent prognostic value in breast carcinoma. In all, our results suggest that separase could be developed into a beneficial biomarker for treatment decisions in breast carcinoma, particularly for patients with luminal subtype.

MATERIALS AND METHODS

Patients and tissue materials. The study comprises tissue specimen obtained from 349 female breast cancer patients. All patients were diagnosed and treated with unilateral invasive breast cancer in Central Hospital of Central Finland, Jyväskylä, Finland, during 1987-1997 (Supplementary material Table 1). All patients were treated with surgical resection or mastectomy with axillary evacuation, radiation and/or adjuvant treatment with antioestrogenic or cytostatic drugs depending on the patients' age, hormone receptor and lymph node status according to the international guidelines for breast cancer treatment at the time of diagnosis (Goldhirsch et al, 2009). No pre-operative adjuvant treatments were administered. Complete clinical data were collected from pathology reports and patient files and registered applying the criteria presented by WHO (Lakhani et al, 2012) and St Gallen International Expert Consensus (Coates et al, 2015). Causes of death were obtained from autopsy reports, death certificates and from the national cancer registry (Statistics Finland, Helsinki, Finland) resulting in up to 22 years and 9 months follow-up period (median 12.4 years).

Separase immunohistochemistry (IHC) of breast cancer tissue was available from each of the 349 patient cases. Tissue materials for IHC and IF were prepared according to standard histology practice, that is, fixed in buffered formalin ( $\mathrm{pH} 7.0)$ and embedded into paraffin blocks. TMAs were prepared by collecting from the representative tumour area of each patient two tissue cores, each $0.6 \mathrm{~mm}$ in diameter. Benign breast epithelium was observed outside carcinomas. In addition, normal breast tissue was obtained from core needle biopsies and breast reductions of healthy young individuals.

Staining methods. IHC was performed on sections cut at $3 \mu \mathrm{m}$. For separase (mouse monoclonal, 6H6, Novus Biologicals, Littleton, CO, USA H00009700-M01, 1:300) we used Lab Vision Autostainer 480 (Thermo-Fisher Scientific, Fremont, CA, USA) with detection performed applying PowerVision + polymer kit with diaminobenzidine as chromogen (DPVB + 110HRP; Immunovision Technologies, Vision Biosystems, Norwell, MA, USA) according to standard protocol. IHC of securin, Ki-67, oestrogen (ER) and progesterone (PR) receptors and HER2 were performed and interpreted as previously described (Gurvits et al, 2016) and HER2-amplification status confirmed with in situ hybridisation (ISH) (Coates et al, 2015).

For double-IF, sections were stained manually using a tyramide signal amplification system for the sequential detection of rabbit and mouse primary antibodies for separase and Ki-67, PHH3, securin, cyclin B1 and Cleaved caspase- 3 as described previously

Table 1. Summary of double-IF ${ }^{\mathrm{a}}$ and triple-IF ${ }^{\mathrm{b}}$ staining methods

\begin{tabular}{|c|c|c|c|c|}
\hline & & & \multicolumn{2}{|l|}{ cyclin B1 } \\
\hline $\begin{array}{l}\text { Antibody } \\
\text { staining }\end{array}$ & $\begin{array}{l}\text { Separase } \\
\text { double- and triple-IF }\end{array}$ & $\begin{array}{l}\text { Securin } \\
\text { double- and triple-IF }\end{array}$ & Double-IF & Triple-IF \\
\hline Type & Mouse monoclonal & Rabbit monoclonal & Rabbit monoclonal & mouse monoclonal \\
\hline Clone & $6 \mathrm{H} 6$ & EPR3240 & Y106 & V152 \\
\hline Source & Novus Biologicals H00009700-M01 & Abcam ab79546 & Abcam ab32053 & MA5-13128 \\
\hline Dilution & $1: 100$ or $1: 1600^{c}$ & 1:500 1h RT & 1:100 1h RT & 1:600 1h RT \\
\hline $\begin{array}{l}\text { Antibody } \\
\text { staining }\end{array}$ & $\begin{array}{l}\text { Ki-67 } \\
\text { double-IF }\end{array}$ & $\begin{array}{l}\mathrm{PHH} 3 \\
\text { double-IF }\end{array}$ & $\begin{array}{l}\text { Cleaved caspase-3 } \\
\text { double-IF }\end{array}$ & \\
\hline Type & Rabbit polyclonal & Rabbit polyclonal & Rabbit monoclonal & \\
\hline Clone & Polyclonal & Polyclonal & D3E9 & \\
\hline Source & Chemicon International AB9260 & Cell Marque 369A-14 & Cell Signaling Technology \#9579 & \\
\hline Dilution & $1: 1000$ & $1: 100$ & $1: 100$ & \\
\hline \multicolumn{5}{|c|}{ 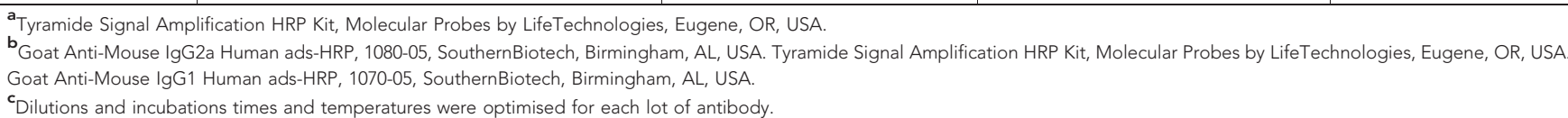 } \\
\hline
\end{tabular}


(Gurvits et al, 2016) (Table 1). Triple-IF was performed as double-IF, but secondary antibodies specific for species and immunoglobulin subtype were used in the detection (Table 1). Control stainings indicated no cross reactions between the used primary and secondary antibodies.

Interpretation of IHC and IF. In IHC of breast tissues, separase expression was observed both in the cytoplasm and in the nucleus of epithelial cells. First, we evaluated separase expression as intensive nuclear precipitates in mitotic cells. In each case, the number of separase-expressing mitotic figures was calculated in sets of one hundred cancer cells (minimum 100 and maximum $3 \times 100$ cancer cells evaluated) and registered as an average fraction (\%) of separase-positive mitoses for each patient (so called mitotic separase). Second, the intensity of the overall separasestaining, so called diffuse separase expression, was evaluated in the cytoplasm and nucleus of cancer cells, and this was registered as negative (score 0 ) or positive ranging from faint to strong $(1+-$ $2+$ ) (so called diffuse separase). Separase expression in the breast carcinoma specimen was evaluated as compared with the expression observed in benign breast epithelium of breast reduction specimen. Based on this comparison, we defined abnormal diffuse separase expression as loss of expression while abnormal mitotic expression was observed as increase in the number of immunopositive cells.

Immunoexpressions for securin and cyclin B1 were observed as combinations of nuclear and cytoplasmic staining and registered as average fractions (\%) of positively staining cancer cells as presented previously (Suzuki et al, 2007; Karra et al, 2012). Interpretations for IHC of ER, PR, and IHC and ISH for HER2 followed previous literature and generally accepted international guidelines (Goldhirsch et al, 2013; Wolff et al, 2014).

All IHC interpretations were performed by an experienced histopathologist (PK). Double- and triple-IF stainings combining separase with Ki-67, PHH3, securin, cyclin B1 and cleaved caspase3 were scanned (Pannoramic Midi II scanner, 3dhistech, using automatic exposure time and ensuring no leakage of fluorescence labels between channels) and co-expressions of the studied proteins were evaluated both in microscopy and in virtually merged photomicrographs.

Statistical analysis. Prognostic analyses were performed separately for mitotic and diffuse separase expressions and for both expression patterns optimal cut points were determined in univariate analyses. Concerning mitotic separase, patient cases were optimally stratified according to absence $v s$ presence $(<1 \%$ vs $\geqslant 1 \%$ ) of separase-positive cancer cell mitoses. For diffuse separase expression (intensities $0-2+$ ), the optimal cut point was identified between negative $v s$ positive expression $(0$ vs $1+-2+)$. The categorisations were supported by morphological observations of separase-IHC in the breast carcinomas and in benign breast tissue. Categorisation of immunoexpressions of securin and cyclin B1 at $10 \%$ of positive cancer cells $(<10 \% v s \geqslant 10 \%)$ were based on previous literature (Karra et al, 2014; Suzuki et al, 2007).

Associations between categorised mitotic and diffuse separase and the other studied markers were analysed by Fisher's exact test and Pearson/Spearman correlation when handled numerically. Survival analyses for mitotic and diffuse separase expressions were first started with univariate analysis for time to breast cancerspecific death and estimated using the Kaplan-Meier technique with Wilcoxon tests. For further analysis involving multiple factors, we evaluated the prognostic value of mitotic and diffuse separase in relation to the established prognosticators of breast cancer that is, nodal status, tumour size, histological grading, and added with immunopositivities for Ki-67 and securin. The final analyses were performed with the help Cox proportional hazard models. Patients with missing data were automatically excluded from the analyses. Differences between categories were quantified by calculating hazard ratio (HRs) with $95 \%$ confidence interval (CI). $P$-values $<0.05$ (two-tailed) were considered statistically significant. The statistical computations were performed using SAS

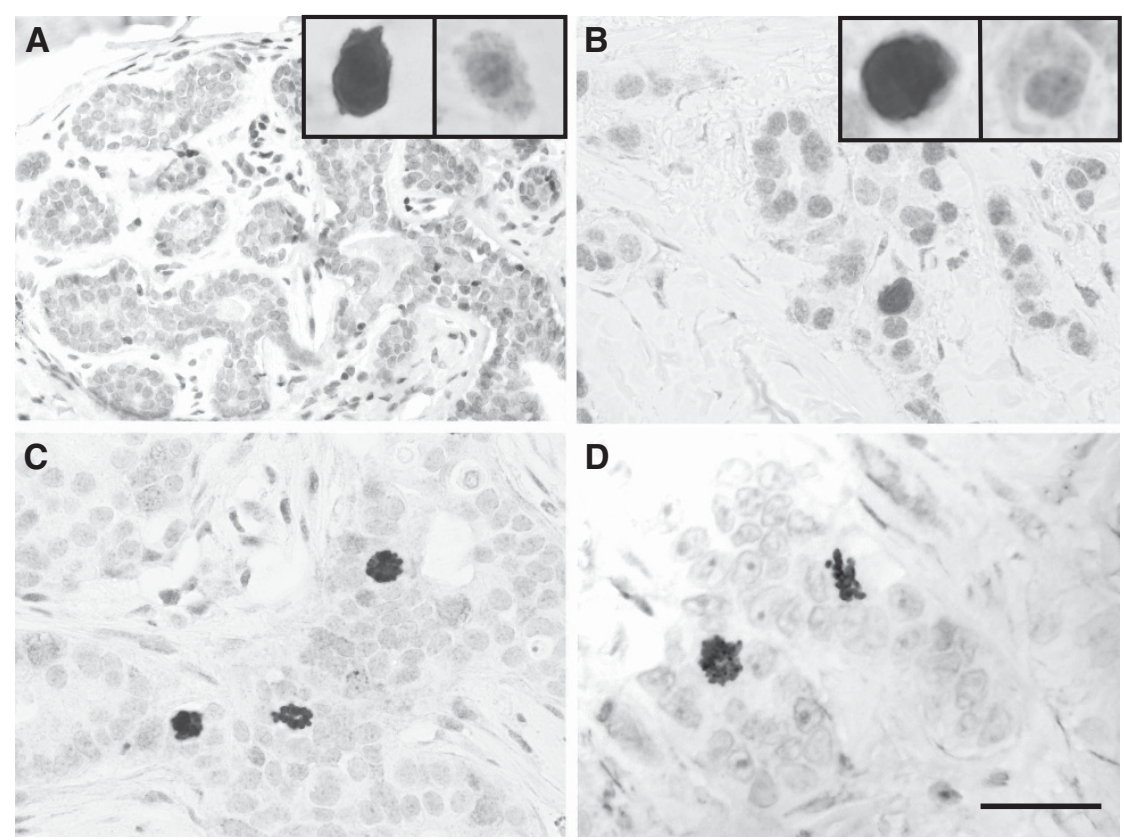

Figure 1. Examples of separase immunoexpression in invasive breast carcinomas and benign breast epithelium. Separase positivity was observed as two distinct staining patterns in the cancer cells, diffuse separase expression in the cytoplasm and nucleus, and mitotic separase. Benign breast tissue showed a clear diffuse separase expression and only single separase-positive mitoses reflecting the low proliferation rate in normal breast epithelium (A). In breast carcinomas representing luminal (B), HER2-amplified (C) and triple-negative breast carcinomas (D), the two staining patterns occurred inversely related so that strong diffuse separase-positivity was associated with low mitotic separase-expression and vice versa. (magnification $\times 400$, space bar $100 \mu \mathrm{m}$ ). A full colour version of this figure is available at the British Journal of Cancer journal online. 
System for Windows, version 9.4 (SAS Institute Inc., Cary, NC, USA).

\section{RESULTS}

In IHC, separase expression in breast carcinoma could be observed as two distinct morphological patterns, first, in mitotic cells and, second, as diffuse expression in the cytoplasm and nucleus of cancer cells (Figure 1). In breast carcinoma, these staining patterns occured inversely related, that is, high mitotic separase expression was usually accompanied with lack of diffuse separase expression, an expression pattern particularly common in carcinomas showing aggressive morphological and clinical features. On the contrary, strong diffuse separase expression was associated with lack of mitotic separase in breast carcinoma cases exhibiting favourable histology and clinical behaviour. Benign breast epithelium showed diffuse nuclear and cytoplasmic separase but mitotic separase in only single cells. In the connective tissue cells of the surrounding stroma, separase expression was occasional and faint.

In Figure 2, separase expression is demonstrated in double-IF stainings with proliferation markers Ki-67, $\mathrm{PHH} 3$ and securin, and apoptosis marker Cleaved caspase-3. In breast carcinoma, separase expression co-localised with $\mathrm{PHH} 3$ in the great majority $(89.2 \%)$ of mitotic cancer cells. Instead, mitotic separase only partly colocalised with Ki-67-positive or securin-positive cancer cells (colocalisation observed in $53.3 \%$ and $62.9 \%$ of cancer cell mitoses, respectively).

In prognostic analyses, mitotic separase was a significant predictor of breast cancer death as demonstrated on basis of Kaplan-Meier curves calculated for disease-specific survival (Figure 3). In univariate approach, the presence of separase- positive mitoses $(\geqslant 1 \%$ of cancer cells) predicted a twofold risk of breast cancer death $(P=0.0004, \mathrm{CI}=1.4-3.0)$. Based on breast cancer-specific survival in the majority $(>75 \%)$ of breast carcinoma cases, we observed an 11.3-year survival difference between patient subgroups detected with and without separase-positive mitotic figures (4.3 and 15.6 years of survival for absence and presence of mitotic separase, respectively) (Figure 3). Kaplan-Meier analyses in subgroups of the intrinsic breast cancer classification also showed prognostic differences between cases detected with and without mitotic separase expression, however, statistically significant prognostic value could only be shown for the luminal subtype (HR 2.2, $P=0.001, \mathrm{CI}=1.4-3.4$ ). Multivariate analyses involving mitotic separase and the established prognosticators of breast carcinoma, tumour size, histological grade and nodal status, are summarised in Table 2 . When securin was added to the multivariate analysis, only securing - and not separase - was associated with statistically significant prognostic value indicating the superior prognostic value of securin over mitotic separase.

We also observed a significant prognostic value for the loss of diffuse separase expression in the breast carcinoma cases (Figure 3). Lack of diffuse separase expression in cancer cells indicated a 1.8 -fold risk of breast cancer death $(P=0.0006$, CI $1.3-$ 2.6) as compared with separase positivity (intensities $1+-2+$ ). Also, we observed a significant survival difference between patient subgroups indicating that the majority $(>75 \%)$ of patients with carcinomas showing diffuse separase expression survived 12.5 years, whereas the patients with loss of diffuse separase expression only 4.9 years after primary diagnosis $(P=0.001)$. In material divided into subgroups of intrinsic classification, only luminal carcinomas showed a statistically significant risk of breast cancer death associated with loss of diffuse separase expression (HR 2.9, $P<0.0001$, CI 1.7-4.9) (Figure 3) whereas no prognostic association could be observed for Her2-amplified or triple-negative

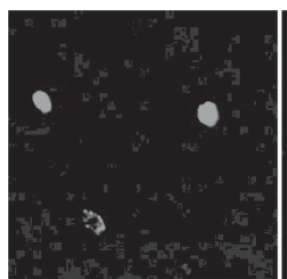

Separase

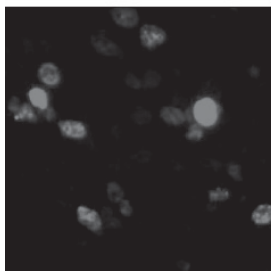

Ki-67

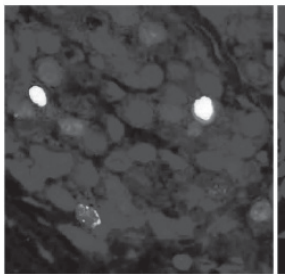

Merge

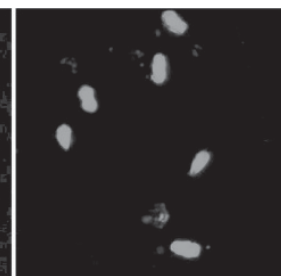

Separase

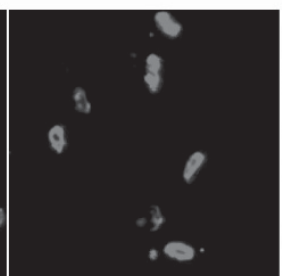

$\mathrm{PHH} 3$

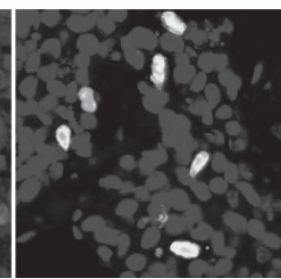

Merge

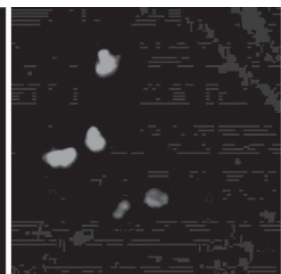

Separase

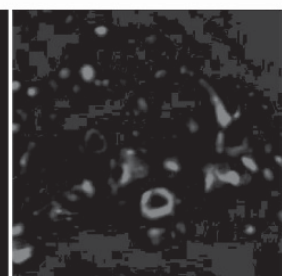

Securin

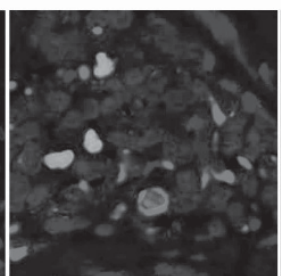

Merge

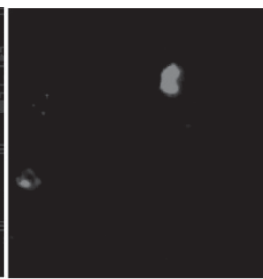

Separase

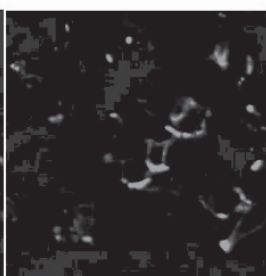

Caspase-3

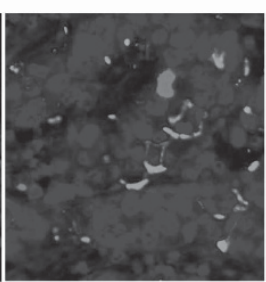

Merge

Figure 2. Immunostaining of separase with established and experimental markers of proliferation in breast carcinoma. Double-IF stainings were performed for combinations of separase (green) with Ki-67, PHH3 and securin (red). In all double-IFs, separase showed co-expression in proliferating cells but the association was most clear between separase and PHH3. Double-IF for separase (red) and Cleaved casepase-3 (green) showed that separase was not expressed in apoptotic cells. (magnification $\times 400$ ). A full colour version of this figure is available at the British Journal of Cancer journal online. 
A

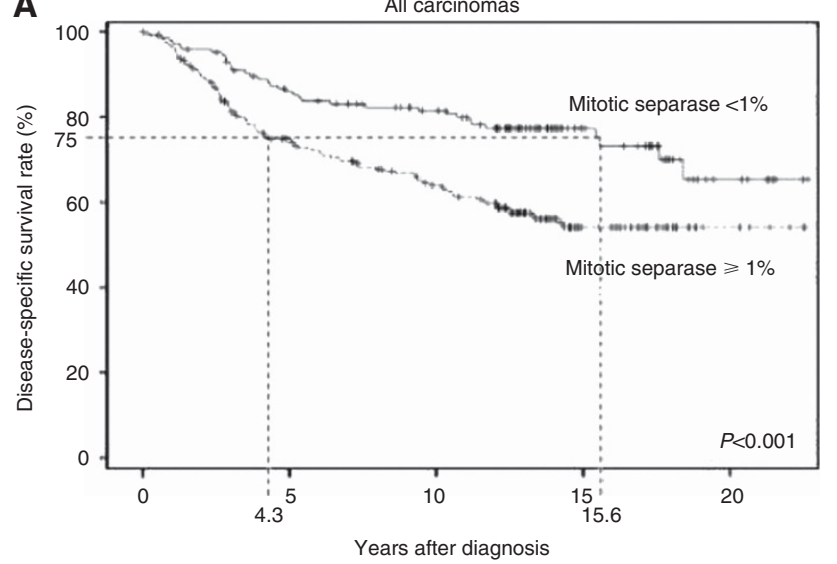

C

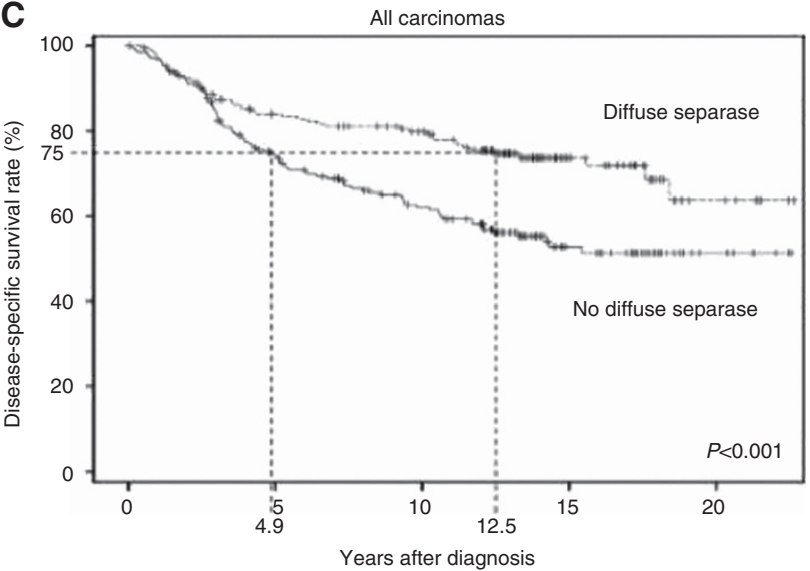

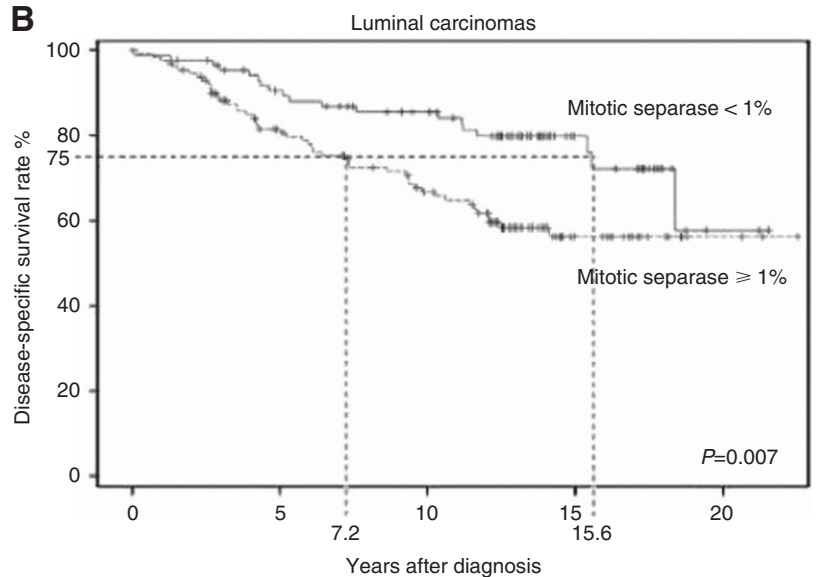

D

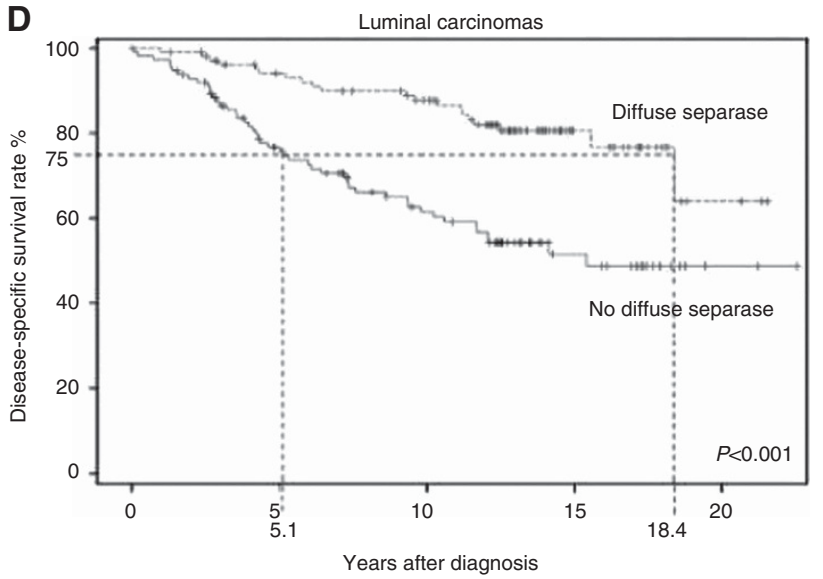

Figure 3. Kaplan-Meier curves of breast cancer-specific survival showing the survival difference of breast carcinoma patients associated with mitotic ( $<1 \%$ vs $\geqslant 1 \%$ of cancer cells) and diffuse separase expression (negative vs positive cancer cells). The figures show survival among all patients $(\mathbf{A}, \mathbf{C})$ and among patients with luminal carcinoma $(\mathbf{B}, \mathbf{D})$. The figures also show survival difference for the majority $(75 \%)$ of patients in both prognostic groups (dashed lines).

Table 2. Multivariate analyses for mitotic and diffuse separase expressions, and the established prognosticators of invasive breast carcinoma

\begin{tabular}{|l|c|c|c|}
\hline & HR & $\boldsymbol{P}$ & Cl \\
\hline Mitotic separase & 1.6 & 0.03 & $1.0-2.5$ \\
\hline Tumour size & 2.1 & 0.001 & $1.3-3.3$ \\
\hline Histological grade & & NS & \\
\hline Nodal status & 3.2 & $<0.0001$ & $2.1-4.8$ \\
\hline Diffuse separase & 1.8 & 0.002 & $1.2-3.8$ \\
\hline Tumour size & 2.2 & 0.0006 & $1.4-3.5$ \\
\hline Histological grade & & NS & \\
\hline Nodal status & 3.2 & $<0.0001$ & $2.1-4.9$ \\
\hline Abbrevions: Cl & & & \\
\hline
\end{tabular}

Abbreviations: $\mathrm{Cl}=95 \%$ confidence interval; $\mathrm{HR}=$ hazard ratio; $\mathrm{NS}=$ no statistical significance; $P=P$-value of Cox's proportional hazard model. The analysis is based on 349 breast carcinoma statistically patients with up to 22-year follow-up. Only analyses with signicificant associations are shown.

${ }^{a}$ Analysis has been performed on material divided into groups with favourable vs unfavourable prognosis as follows: Mitotic separase comparing low vs high expression $(<1 \%$ vs $\geqslant 1 \%$ separase-positive mitoses in cancer cells). Diffuse separase comparing positive (intensity $1+-2+$ ) vs negative $(0)$ expression in carcinoma cells. Tumour size stratified into small vs large ( $\leqslant 2 \mathrm{~cm}$ vs $>2 \mathrm{~cm}$ in diameter). Nodal status startified into axillary lymph node negative vs positive. Histological grade compared between 1-2 and 3 .

carcinomas (TNBC). In multivariate analysis involving the established clinical prognosticators of breast cancer, loss of separase expression showed independent prognostic value along with nodal status and tumour size $(\mathrm{HR}=1.8, P=0.002$, CI $1.3-$ 2.6) (Table 2). Finally, in a multivariate analysis involving both diffuse and mitotic separase, securin, Ki-67 and the established clinical prognosticators, loss of diffuse separase expression still remained an independent prognosticator for breast cancer death (HR 1.6, $P=0.02$, CI 1.1-2.4).

Finally, we wanted to contemplate the possible interactions between separase, securin and cyclin B1 in driving cancer cell proliferation in breast carcinoma. Based on statistical analysis of separase-IHC, mitotic separase was strongly associated with high expression levels of both securin $(P<0.0001)$ and cyclin B1 $(P<0.0001)$. To evaluate their expressions at the cellular level and make conclusions on their associations and possible mutual regulation in mitosis, we prepared double- and triple-IF stainings to demonstrate their co-expression in cells of breast carcinoma and benign breast epithelium. Double-IFs of breast carcinoma showed that separase-positive cells typically co-expressed cyclin B1 but not securin (Figure 4). This expression pattern resembles the one seen in normal breast epithelium, although owing to the low mitotic rate, the occurrence of positive cells in normal epithelium was naturally very rare. Instead, some carcinomas showed aberrant expression where cancer cells either co-expressed separase and securin or, alternatively, showed separase-positivity alone. These aberrant expression patterns were also verified in triple-IFs showing either the simultaneous expressions of the three markers or only separase expression in the same cancer cell (Figure 4). 
A

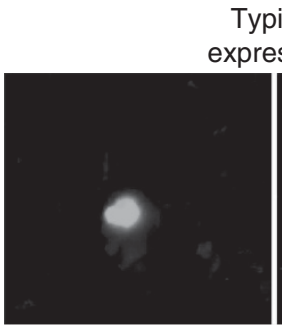

Separase

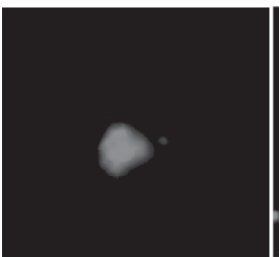

Cyclin B1

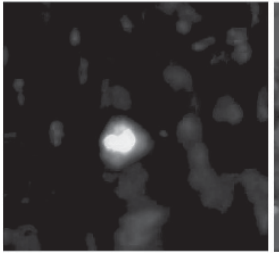

Merge
Typical

xpression

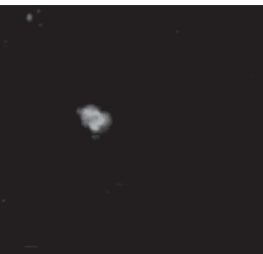

Separase

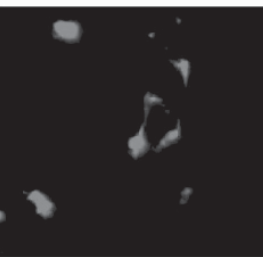

Securin

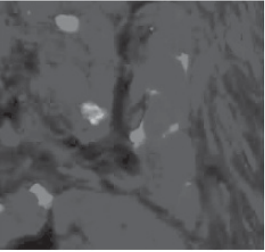

Merge

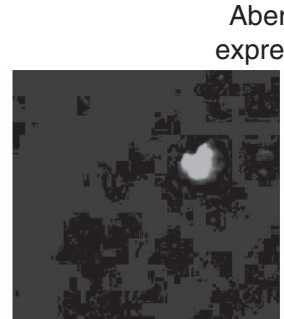

Separase

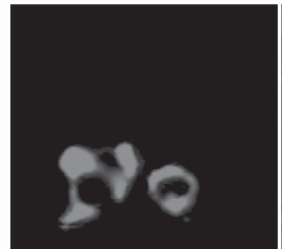

Cyclin B1

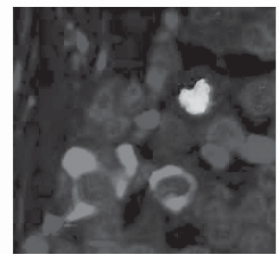

Merge
Aberrant

expression

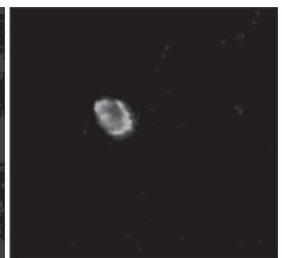

Separase

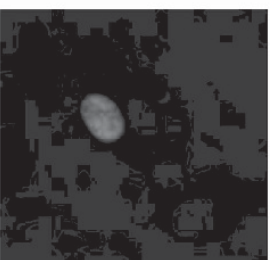

Securin

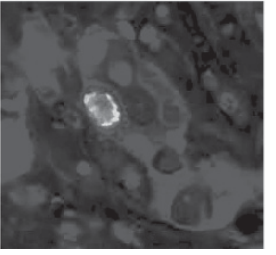

Merge

B

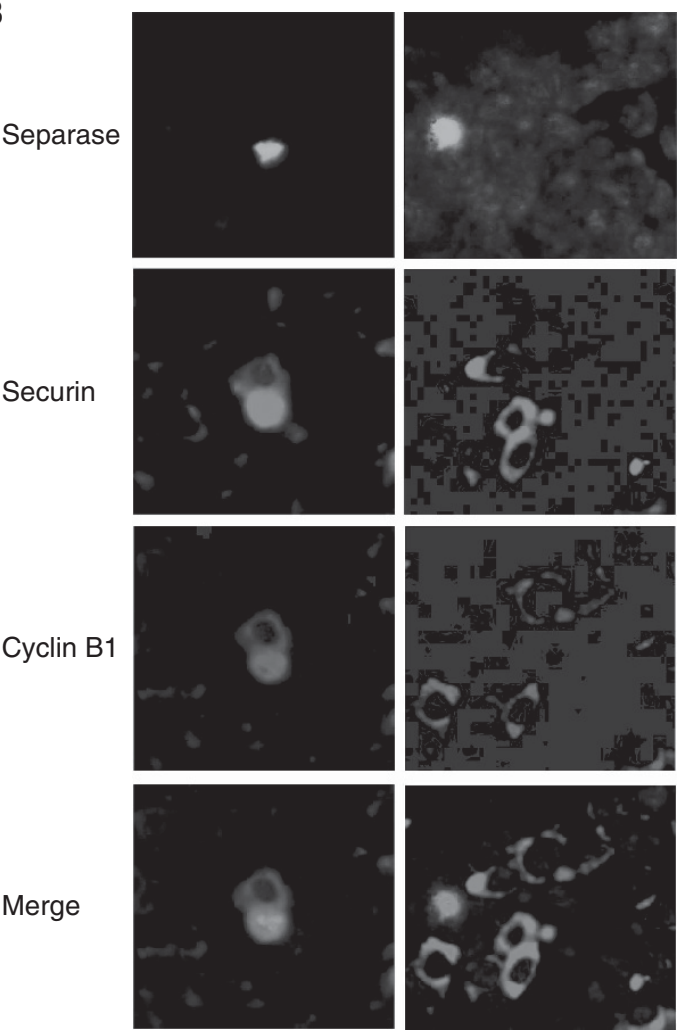

Figure 4. Double- and triple-IFs demonstrate the co-localisation and distribution of separase with securin and cyclin B1 in invasive breast carcinomas. The co-expression patterns for separase and securin, and separase and cyclin B1 were similar in benign breast epithelium and in the majority of carcinoma cells that is, separase-positive cells typically co-expressed cyclin B1 but not securin (A). In some carcinomas of aggressive morphology, however, we observed an aberrant expression pattern where cancer cells either co-expressed separase and securin or, alternatively, showed only separase positivity (A). These aberrant expression patterns were also verified in triple-IFs showing cancer cells with either simultaneous expression of the three markers or separase expression alone (B). (magnification $\times 400$ ). A full colour version of this figure is available at the British Journal of Cancer journal online. 


\section{DISCUSSION}

Separase-IHC showed distinct prognostic value in our analysis involving 349 invasive breast carcinoma patients with a maximum of $>22$-year follow-up. The prognostic value was obvious for both expression patterns observed for separase, that is, mitotic and diffuse separase. In our material, mitotic separase predicted a doubled risk of breast cancer death $(P=0.0004)$ and predicted 11.3-year survival difference as calculated comparing the majority $(>75 \%)$ of patients with and without separase-expressing cancer cell mitoses. Interestingly, the impact of diffuse separase expression on patient outcome was inversed, that is, lack of diffuse separase indicated 1.8 -fold risk of breast cancer death $(P=0.0006)$ as compared with diffuse separase-positivity. Again, a significant survival difference could be detected so that the majority $(>75 \%)$ of patients lacking diffuse separase expression in their breast carcinomas lived 7.6 years longer than the patients with carcinomas showing diffuse separase positivity. Both mitotic and diffuse separase expressions also predicted significantly impaired disease-specific outcome among the subgroup of luminal breast carcinomas (HR 1.3, $P=0.02$ and HR 2.9, $P<0.0001$, respectively). Instead, we could not detect prognostic value in TNBC or HER2amplified carcinomas, possibly due to the small number of patients in these subgroups. In multivariate analyses, both mitotic separase and loss of diffuse separase expression showed independent prognostic value among all breast carcinomas (Table 2). In summary, our results suggest that patients with high mitotic separase have unfavourable prognosis, whereas patients with diffuse separase have survival advantages. High rate of mitotic separase may be seen to reflect high proliferative activity, which is a known feature of aggressive behavior in malignant disease, especially as separase overexpression has been shown to promote aneuploid cell divisions and genetic heterogeneity (Zhang and Pati, 2017). The association of diffuse separase with favourable prognosis remains unsettled but has been suggested to reflect the activity of separase where auto-cleavage of separase may have a role (Zhang and Pati, 2017). Separase protein overexpression has been demonstrated in several human malignancies, including breast carcinomas, osteosarcomas, glioblastomas and chronic myeloid leukaemias (Meyer et al, 2009; Patel and Gordon, 2009; Mukherjee et al, 2014a). Previously, separase expression has been associated with relapse, metastasis and/or survival in prostate cancers and glioblastoma (Meyer et al, 2009; Mukherjee et al, 2014a). In breast carcinoma, separase RNA levels have been associated with metastatic disease, especially for Luminal B subtype (Meyer et al, 2009; Finetti et al, 2014). To our knowledge, there are no previous reports on the association of separase to breast cancer survival. Also separase gene expression, as evaluated in a metaselection, has been associated with carcinogenesis and prognosis in several solid tumours (Rouam et al, 2010).

Separase is a multifunctional protein that has been under intensive research as its discovery (Uhlmann et al, 2000). The functions of separase in oncogenesis and tumour progression as well as in both mitotic and meiotic divisions have been well established (Zhang and Pati, 2017). When the sister kinetochores are bioriented in metaphase, separase participates in the proteolytical cleavage of cohesion, which ultimately triggers the separation of the sister chromatids. This transition from metaphase to anaphase is controlled by the APC/C (Primorac and Musacchio, 2013). In previous literature, the spatial localisation of separase has been reported to vary with the progression of the cell cycle so that in interphase separase is localised in the cytoplasm whereas during mitosis it accumulates in the nucleus and associates with chromosomes (Zhang and Pati, 2017). Abnormal function of separase has been associated with aneuploidy and genetic instability inducing subsequent tumourigenesis. Transgenic mice expressing the MMTV-separase cDNA have been shown to develop aneuploidy and highly aggressive and heterogeneous mammary adenocarcinomas of both luminal and basal subtypes (Mukherjee et al, 2014b). Both cyclin B1 and securin are known to essentially regulate the separase-dependent events in metaphaseanaphase transition (Chang and Barford, 2014). Previous literature has addressed mitotic slippage, that is, the escape of mitosisincompetent cells from mitotic arrest, as one possible mechanism by which the normally controlled sequence of degradation of cyclin B1 and/or securin may be overridden at the metaphase-anaphase transition (Toda et al, 2012). However, there are numerous hypotheses on the role of separase in aberrant cell expression (Kamenz and Hauf, 2016). In our double-IFs, we saw different expression patterns for separase, securin and cyclin B1. Typically, separase-positive cells co-expressed cyclin B1 but not securin, a finding consistent with the expedient pattern of normal separasedependent cell cycle control. However, as observed in our triple-IFs at a single-cell level, an aberrant expression pattern seen in some carcinomas with aggressive morphology may reflect abnormal cell cycle control driving aneuploidy and chromosomal instability, both common features in high-grade breast carcinomas and especially TNBC. In mouse mammary epithelium, the transcriptional regulation of separase expression has been shown to be facilitated by loss of p53 and induced by ER and PR (Pati et al, 2004). These observations are in line with the general understanding that misregulated sister chromatid segregation favours aneuploidy and drives proliferation in aggressive breast carcinomas (Meyer et al, 2009; Yadav et al, 2015).

In malignant disease, separase has been described showing different expression patterns and subcellular localisations reflecting the numerous roles of separase in oncogenesis (Zhang and Pati, 2017). Previously, not only the total cellular level of separase but also its subcellular expression patterns have been reported to vary between normal tissue and different stages of malignancy. Previously in literature, however, only nuclear separase has been associated with tumour status in human carcinomas, including breast cancer (Meyer et al, 2009). The exact expression patterns of separase and their prognostic associations have not previously been systematically described in breast cancer. Interestingly, we observed a prognostic impact associated with translocation of separase from the cytoplasm to the mitotic nucleus. As a new finding, diffuse separase observed in the cancer cells independently predicted breast cancer outcome in our material. According to experiments in budding yeast, securin is responsible for separase being translocated into the nucleus and, in DNA damage, prevents the cell from progressing to anaphase (Hornig et al, 2002). It has been proposed that separase might be important in DNA damage repair (Nagao et al, 2004). However, the connection of overexpression and subcellular localisation of separase to tumour formation and/or progression is not yet understood. These observations suggest the hypothesis that misregulation of sister chromatid segregation by hyperactive separase induces chromosomal instability and aneuploidy, common features in TNBC (AlEjeh et al, 2014; Mukherjee et al, 2014b; Yadav et al, 2015). Our findings from double- and triple-IFs also demonstrate the abnormal regulation of separase and emphasise the independent role of separase in driving proliferation.

Currently, molecular subtyping is the basis for breast cancer diagnostics and treatment, proliferation being one of the routine indicators for disease aggressiveness. In spite of the advent of molecular testing, intrinsic classification based on surrogate markers, including proliferation, is still the basis of breast cancer classification and treatment decisions, particularly in case of the luminal B subtype (Prat et al, 2011; Lakhani et al, 2012). Moreover, traditional histological grade and the Nottingham Grading Index are considered among the most accurate methods to assess the biological characteristics and patient survival in breast cancer 
(Rakha et al, 2010; Schwartz et al, 2014). Both surrogate criteria of the intrinsic classification and histological grading, however, involve mitotic counting, which is considered a laborious and time consuming method (Del Sordo et al, 2017). Also, the routine proliferation markers of clinical pathology, that is, Ki-67 and PHH3 IHC do not provide optimal prognostic information for breast cancer. Ki67-IHC is often considered difficult to interpret due to its heterogeneity and varying intensity (Jalava et al, 2006). The interpretation of PHH3-IHC is a more solid but the detection method is particularly susceptible to poor fixation. In our experience, detection and interpretation of separase-positive mitoses is easy and reproducible. Based on uni- and multivariate survival analyses, our results also demonstrate the superior prognostic value of separase to histological grading and Ki-67.

In summary, our findings from 349 human breast carcinomas with maximum of $>22$ years of follow-up show that separase is a potential prognosticator with independent value in identifying disease-specific survival. As a proliferation marker, separase-IHC is well applicable to clinical routine and shows practical and prognostic value over $\mathrm{PHH} 3$ and Ki-67. Our observations on double- and triple-IFs suggest that aberrant expression patterns of separase and its regulating proteins, securin and cyclin B1, identify patients with particularly aggressive subtypes of breast carcinoma. In all, we suggest that separase could be developed into a beneficial biomarker for treatment decisions and a potential therapeutic target for breast carcinoma.

\section{ACKNOWLEDGEMENTS}

The authors thank Mrs Sinikka Collanus, PhD Markus Peurla and Mr Jaakko Liippo, Institute of Biomedicine, University of Turku, Turku, Finland. The work has been approved by the ethical committees of Turku University Hospital and Central Hospital of Central Finland, Turku, Finland (permit numbers 0286/2002, 7765-2002). The presented ethical permissions include informed patient consents.

\section{CONFLICT OF INTEREST}

The authors declare no conflict of interest.

\section{REFERENCES}

Al-Ejeh F, Simpson PT, Sanus JM, Klein K, Kalimutho M, Shi W, Miranda M, Kutasovic J, Raghavendra A, Madore J, Reid L, Krause L,

Chenevix-Trench G, Lakhani SR, Khanna KK (2014) Meta-analysis of the global gene expression profile of triple-negative breast cancer identifies genes for the prognostication and treatment of aggressive breast cancer. Oncogenesis 21(3): e100.

Chang L, Barford D (2014) Insights into the anaphase-promoting complex: a molecular machine that regulates mitosis. Curr Opin Struct Biol 29: 1-9.

Coates AS, Winer EP, Goldhirsch A, Gelber RD, Gnant M, Piccart-Gebhart M, Thürlimann B, Senn HJ. Panel Members (2015) Tailoring therapiesimproving the management of early breast cancer: St Galen International Expert Consensus on the Primary Therapy of Early Breast Cancer 2015. Ann Oncol 26: 1533-1546.

Del Sordo R, Bellezza G, Ferri I, Pireddu A, Colella R, Sidoni A (2017) Mitotic index matter: how to improve the assessment of mitosis in order to better classify G2 breast cancer and luminal A category. Histol Histopathol 23: 11891.

Finetti P, Guille A, Adelaide J, Birnbaum D, Chaffanet M, Bertucci F (2014) ESPL1 is a candidate oncogene of luminal B breast cancers. Breast Cancer Res Treat 147: 51-59.

Goldhirsch A, Ingle JN, Gelber RD, Coates AS, Thürlimann B, Senn HJ. Panel members (2009) Thresholds for therapies: highlights of the St Gallen
International Expert Consensus on the primary therapy of early breast cancer. Ann Oncol 20: 1319-1329.

Goldhirsch A, Winer EP, Coates AS, Gelber RD, Piccart-Gebhart M, Thurlimann B, Senn HJ. Panel members (2013) Personalizing the treatment of women with early breast cancer: highlights of the St Gallen International Expert Consensus on the Primary Therapy of Early Breast Cancer 2013. Ann Oncol 24: 2206-2223.

Gurvits N, Repo H, Löyttyniemi E, Nykänen M, Anttinen J, Kuopio T, Talvinen K, Kronqvist P (2016) Prognostic implications of securin expression and sub-cellular localization in human breast cancer. Cell Oncol 39: 319-331.

Haaß W, Stehle M, Nittka S, Giehl M, Schrotz-King P, Fabarius A, Hofmann WK, Seifarth W (2012) The proteolytic activity of separase in BCR-ABL-positive cells is increased by imatinib. PLoS One 7: e42863.

Hellmuth S, Rata S, Brown A, Heidmann S, Novak B, Stemmann O (2015) Human chromosome segregation involves multi-layered regulation of separase by the peptidyl-prolyl-isomerase Pin1. Mol Cell 58: 495-506.

Holland AJ, Taylor SS (2006) Cyclin-B1-mediated inhibition of excess separase is required for timely chromosome disjunction. J Cell Sci 119: 3325-3336.

Hornig NC, Knowles PP, McDonald NQ, Uhlmann F (2002) The dual mechanism of separase regulation by securin. Curr Biol 12: 973-982.

Jalava P, Kuopio T, Juntti-Patinen L, Kotkansalo T, Kronqvist P, Collan Y (2006) Ki-67 immunohistochemistry: a valuable marker in prognostication but with a risk of misclassification: proliferation subgroups formed based on Ki67 immunoreactivity and standardized mitotic index. Histopathology 48: 674-682.

Kamenz J, Hauf S (2016) Time to split up: dynamics of chromosome separation. TICB 27: 42-54.

Karra H, Pitkänen R, Nykänen M, Talvinen K, Kuopio T, Söderström M, Kronqvist P (2012) Securin predicts aneuploidy and survival in breast cancer. Histopathology 60: 586-596.

Karra H, Repo H, Ahonen I, Löyttyniemi E, Pitkänen R, Lintunen M, Kuopio T, Söderström M, Kronqvist P (2014) Cdc20 and securin overexpression predict short-term breast cancer survival. Br J Cancer 110: 2905-2913.

Lakhani SR, Ellis IO, Schnitt SJ, Tan PH, van de Vijver MJ (eds.) (2012) WHO Classification of Tumours of the Breast. pp 10-11. IARC: Lyon.

Meadows JC, Millar JB (2015) Sharpening the anaphase switch. Biochem Soc Trans 43: 19-22.

Meyer R, Fofanov V, Panigrahi A, Merchant F, Zhang N, Pati D (2009) Overexpression and mislocalization of the chromosomal segregation protein separase in multiple human cancers. Clin Cancer Res 15: 2703-2710.

Mukherjee M, Byrd T, Brawley VS, Bielamowicz K, Li XN, Merchant F, Maitra S, Sumazin P, Fuller G, Kew Y, Sun D, Powell SZ, Ahmed N, Zhang N, Pati D (2014a) Overexpression and constitutive nuclear localization of cohesin protease Separase protein correlates with high incidence of relapse and reduced overall survival in glioblastoma multiforme. J Neurooncol 119: 27-35.

Mukherjee M, Ge G, Zhang N, Edwards DG, Sumazin P, Sharan SK, Rao PH, Medina D, Pati D (2014b) MMTV-Espl1 transgenic mice develop aneuploid, estrogen receptor alpha (ERa)-positive mammary adenocarcinomas. Oncogene 33: 5511-5522.

Mora-Santos M, Limón-Mortés MC, Giráldez S, Herrero-Ruiz J, Sáez C, Japón MÁ, Tortolero M, Romero F (2011) Glycogen synthase kinase-3beta (GSK3beta) negatively regulates PTTG1/human securin protein stability, and GSK3beta inactivation correlates with securin accumulation in breast tumors. J Biol Chem 286: 30047-30056.

Nagao K, Adachi Y, Yanagida M (2004) Separase-mediated cleavage of cohesion at interphase is required for DNA repair. Nature 430: 1044-1048.

Patel H, Gordon MY (2009) Abnormal centrosome-centriole cycle in chronic myeloid leukaemia? Br J Haematol 146: 408-417.

Pati D (2008) Oncogenic activity of separase. Cell Cycle 7: 3481-3482.

Pati D, Haddad BR, Haegele A, Thompson H, Kittrell FS, Shepard A, Montagna C, Zhang N, Ge G, Otta K, McCarthy M, Ullrich RL, Medina D (2004) Hormone-induced chromosomal instability in p53-null mammary epithelium. Cancer Res 64: 5608-5616.

Primorac I, Musacchio A (2013) Panta rhei: the APC/C at steady state. J Cell Biol 201: 177-189.

Prat A, Ellis MJ, Perou CM (2011) Practical implications of gene-expressionbased assays for breast oncologists. Nat Rev Clin Oncol 9: 48-57. 
Rakha EA, Reis-Filho JS, Baehner F, Dabbs DJ, Decker T, Eusebi V, Fox SB, Ichihara S, Jacquemier J, Lakhani SR, Palacios J, Richardson AL, Schnitt SJ, Schmitt FC, Tan PH, Tse GM, Badve S, Ellis IO (2010) Breast cancer prognostic classification in the molecular era: the role of histological grade. Breast Cancer Res 12: 207.

Rouam S, Moreau T, Broët P (2010) Identifying common prognostic factors in genomic cancer studies: a novel index for censored outcomes. $B M C$ Bioinformatics 11: 150.

Schöckel L, Möckel M, Mayer B, Boos D, Stemmann O (2011) Cleavage of cohesin rings coordinates the separation of centrioles and chromatids. Nat Cell Biol 13: 966-972.

Schwartz AM, Henson DE, Chen D, Rajamarthandan S (2014) Histologic grade remains a prognostic factor for breast cancer regardless of the number of positive lymph nodes and tumor size: a study of 161708 cases of breast cancer from the SEER Program. Arch Pathol Lab Med 138: 1048-1052.

Shindo N, Kumada K, Hirota T (2012) Separase sensor reveals dual roles for separase coordinating cohesin cleavage and cdk1 inhibition. Dev Cell 23: $112-123$.

Sun Y, Kucej M, Fan HY, Yu H, Sun QY, Zou H (2009) Separase is recruited to mitotic chromosomes to dissolve sister chromatid cohesion in a DNAdependent manner. Cell 137: 123-132.

Suzuki T, Urano T, Miki Y, Moriya T, Akahira J, Ishida T, Horie K, Inoue S, Sasano H (2007) Nuclear cyclin B1 in human breast carcinoma as a potent prognostic factor. Cancer Sci 98: 644-651.

Toda K, Naito K, Mase S, Ueno M, Uritani M, Yamamoto A, Ushimaru T (2012) APC/C-Cdh1-dependent anaphase and telophase progression during mitotic slippage. Cell Div 7: 4.

Uhlmann F, Wernic D, Poupart MA, Koonin EV, Nasmyth K (2000) Cleavage of cohesin by the $\mathrm{CD}$ clan protease separin triggers anaphase in yeast. Cell 103: $375-386$.
Waizenegger I, Gimenez-Abian JF, Wernic D, Peters JM (2002) Regulation of human separase by securin binding and autocleavage. Curr Biol 12: 1368-1378.

Wolff AC, Hammond MEH, Hicks DG, Dowsett M, McShane LM, Allison KH, Allred DC, Bartlett JM, Bilous M, Fitzgibbons P, Hanna W, Jenkins RB, Mangu PB, Paik S, Perez EA, Press MF, Spears PA, Vance GH, Viale G, Hayes DF. American Society of Clinical Oncology; College of American Pathologists (2014) Recommendations for human epidermal growth factor receptor 2 testing in breast cancer: American Society of Clinical Oncology/College of American Pathologists clinical practice guideline update. Arch Pathol Lab Med 138 : 241-256.

Yadav BS, Chanana P, Jhamb S (2015) Biomarkers in triple negative breast cancer: a review. World J Clin Oncol 6: 252-263.

Zhang N, Ge G, Meyer R, Basu D, Sethi S, Pradhan S, Zhao Yi-Jue Li X-N, Cai WW, El-Naggar AK, Baladandayuthapani V, Kittrell FS, Rao P, Medina D, Pati D (2008) Overexpression of Separase induces aneuploidy and mammary tumourigenesis. PNAS 105: 13033-13038.

Zhang N, Pati D (2017) Biology and insights into the role of cohesin protease separase in human malignancies. Biol Rev Camb Philos Soc; doi:10.1111/ brv. 12321

Zhang N, Scorsone K, Ge G, Kaffes CC, Dobrolecki LE, Mukherjee M, Lewis MT, Berg S, Stephan CC, Pati D (2014) Identification and characterization of separase inhibitors (sepins) for cancer therapy. J Biomol Screen 19: 878-889.

This work is published under the standard license to publish agreement. After 12 months the work will become freely available and the license terms will switch to a Creative Commons AttributionNonCommercial-Share Alike 4.0 Unported License.

Supplementary Information accompanies this paper on British Journal of Cancer website (http://www.nature.com/bjc) 